\section{Registro de paro cardiorrespiratorio en un hospital universitario en el período 2006-2017}

\section{Registry of Perioperative cardiac arrest in a clinical hospital in the period 2006-2017}

\section{Señor Editor:}

He leído con profundo interés el trabajo de la Dra. Aguirre y cols ${ }^{1}$, el cual aporta rica información cuyo conocimiento será de vital importancia para la comunidad anestesiológica nacional. Existen, sin embargo, algunos alcances y dudas que podrían plantearse.

No se definió a priori la edad de la población incluida en el estudio (se asume que son adultos y niños puesto que el rango etario parte desde desde un año). Las implicancias son diferentes dependiendo de si la población estudiada es adulta o pediátrica ${ }^{2}$.

¿Existía alguna relación entre la ocurrencia del evento y el estado físico del paciente según la clasificación de la American Society of Anesthesiologists, (ASA)? Dicha escala ha sido recientemente incluida en un instrumento de categorización del riesgo en pacientes quirúrgicos ${ }^{3}$.

Sería interesante conocer por separado el número total de pacientes sometidos a cirugía de urgencia y electiva en el período estudiado para así determinar la incidencia del evento en cada grupo. La cirugía de urgencia es un factor reconocido de riesgo perioperatorio ${ }^{4}$.

Se resalta que la condición pre-existente más frecuentemente asociada fue la hipotensión e hipo-perfusión, dicho estado, por lógica solo puede haberse presentado en pacientes sometidos a cirugía de urgencia, lo cual plantea la interrogante de cuál fue la condición más frecuentemente asociada al evento estudiado en cirugía electiva.

En la clasificación de las causas de paro cardíaco (Tabla 4); parece confuso el incluir la hipovolemia (de origen no hemorrágico), como causa quirúrgica y el trasplante como causa médica. Entre las causas anestésicas parecería superfluo separar el ítem " desconexión del circuito anestésico" de la categoría "hipoxia".

Si bien el objetivo del estudio es descriptivo, es inevitable preguntarse si hubo algún atisbo o sospecha de cuales variables influenciaron la mortalidad, el pronóstico neurológico y el tiempo de sobrevida; ¿Edad?, ¿Género?, ¿Patologías asociadas?
Finalmente se sugiere que la causa más probable de paro cardíaco durante anestesia espinal fue la ocurrencia de un reflejo de Bezold-Jarisch. Para corroborarlo sería importante conocer si hubo bradicardia previo al evento. En este mismo sentido, ¿no se deberían también considerar otras causas como la hipovolemia no reconocida ${ }^{5}$ la hipoventilación e hipoxemia secundaria al uso de sedantes? ${ }^{6}$. Para finalizar, solo resta agradecer a los autores de este estudio que sin duda contribuirá a la mejor comprensión y prevención de un evento de máxima gravedad.

Dagoberto Ojeda Dinamarca ${ }^{1,2}$ ${ }^{1}$ Anestesiólogo Clínica Dávila. Santiago, Chile. ${ }^{2}$ Magister Bioestadística.

\section{Referencias}

1. Aguirre MM, Mayanz S, Blanch A, Aranibar H, Salazar A, Roizen G, et al. Registro de paro cardiorrespiratorio en un hospital universitario en el período 2006-2017. Rev Med Chile 2019; 147: 34-40.

2. Braz LG, Módolo NS, do Nascimento P Jr, Bruschi BA, Castiglia YM, Ganem EM, et al. Perioperative cardiac arrest: a study of 53,718 anaesthetics over 9 years from a brazilian teaching hospital. Br J Anaesth 2006; 96: 569-75.

3. Protpaka KL, Simpson JC, Smith NCE, Moonesinghe SR. Development and Validation of the surgical outcome risk tool (SORT). Br J Surg 2014; 101: 1774-83.

4. Moonesinghe SR, Mythen MG, Das P, Rowan KM, Grocott MPW. Risk stratification tools for predicting morbidity and mortality in adults patiens undergoing major surgery :qualitative systematic review. Anesthesiology 2013; 119: 959-81.

5. Kumari A, Gupta R, Bajwa SJS, Singh A. Unanticipated cardiac arrest under spinal anesthesia: An unavoidable mystery with review of the current literature. Anesth Essays Res 2014; 8: 99-102.

6. Barreiro G, Van Zundert A, Al-Shaikh A. Unexpected cardiac arrest in spinal anesthesia. Acta Anaesth Belg 2006; 57: 365-70.

Correspondencia a:

Dr. Dagoberto Ojeda D

Servicio de Anestesiología Clínica Dávila,

Av. Recoleta 464, Recoleta, Edifico D-3 $3^{\text {er }}$ piso, Santiago, Chile.

eojedadinamarca@gmail.com 
Los autores aludidos ofrecieron la siguiente réplica:

\section{Sr. Editor:}

En respuesta a las observaciones realizadas por el Dr. Dagoberto Ojeda, manifestamos lo siguiente:

En cuanto a la edad de los pacientes, como criterio de inclusión se definió todo paciente que requiriera compresiones torácicas y/o desfibrilación desde la entrada al área de pabellones quirúrgicos o la sala de procedimiento fuera de pabellón, hasta el alta desde la unidad posanestésica. Los criterios de exclusión fueron eventos ocurridos en pacientes sometidos a cirugía cardíaca y los casos con orden de no reanimar. Dada esta definición de criterios, la edad no constituyó una variable que condicionara la inclusión, homologando el método de otros autores ${ }^{1-3}$. Registramos 6 pacientes pediátricos. Hubo dos asistolias de rápida resolución atribuidas a reflejo vagal gatillado por estímulo orofaríngeo en adenoidectomías en niños sanos, un evento de casusa eléctrica en niño portador de miocardiopatía, y tres eventos de causa hipóxica.

La razón por la que no incluimos la clasificación de la American Society of Anesthesiologists (ASA) obedece a que en este estudio utilizamos una pauta de recolección de datos al estilo Utstein modificada, la cual tiende a estandarizar los registros de modo de que sean comparables y esta no considera la clasificación mencionada, por lo que no fue considerada ${ }^{4}$.

En nuestro estudio, las cirugías electivas fueron $51,25 \%$ y las de urgencia $48,75 \%$. En los registros electrónicos de nuestro hospital no se consigna si el paciente que entra a pabellón es de urgencia o electivo, sólo queda registrado el código quirúrgico, por lo que no se cuenta con el denominador para las cirugías de urgencia.

Con relación a la causa preexistente más frecuentemente encontrada en cirugía electiva, esta fue la enfermedad tumoral metastásica o hematológica, presente en 10 de los eventos, seguidos por arritmias y diabetes en 5; respectivamente. A su vez, la principal causa de paro cardiorrespiratorio en este grupo mayoritario fue la hemorragia intraoperatoria en 4 casos. Otras causas fueron: síndrome coronario agudo intraoperatorio, arritmia durante instalación de catéter de arteria pulmonar, tromboembolismo pulmonar masivo intraoperatorio, mecánico (drenaje aspirativo en contacto con aurícula derecha), un caso respectivamente, e hipovolemia relativa más efecto de drogas anestésicas en dos casos. Hacemos la salvedad de que no consideramos que algunas de las condiciones preexistentes sean por defecto excluyentes de pacientes electivos o de urgencia. Por ejemplo, un paciente electivo podría ingresar hipovolémico a pabellón después de una preparación de colon sin reposición adecuada de fluidos.

En cuanto a las causas del paro, efectivamente hay un error de edición/impresión de la tabla. La causa "hipovolemia" corresponde al grupo de las complicaciones preoperatorias. Las causas quirúrgicas intraoperatorias sólo fueron la hemorragia, embolía aérea y otros. Se decidió categorizar el "trasplante" como causa médica, puesto que las condiciones que finalmente contribuyen al paro cardiorrespiratorio en este escenario constituyen en conjunto una causalidad multifactorial, donde es difícil muchas veces identificar el principal factor determinante. Entre estos están la hiperkalemia, hipotermia, anemia, acidosis, hipocalcemia y fenómenos de reperfusión, todas condiciones que consideramos más bien médicas, en tanto perturban gravemente el equilibrio del medio interno. En todo caso, sólo responde a una categorización arbitraria con fines de orden para poder obtener conclusiones útiles, tal como lo han considerado otros autores ${ }^{2}$.

En relación a considerar la desconexión separada de la hipoxia como causa anestésica, concordamos con la observación de que obviamente la causa final del paro cardiorrespiratorio en este caso es la hipoxia, sin embargo, creemos que la connotación es diferente y merece ser abordada en forma especial, ya que las medidas necesarias para evitarla tienen mucho más que ver con aquellas de orden sistémico de prevención de errores y de seguridad, más que con estrategias eminentemente clínicas como ocurre en los otros casos categorizados como "hipoxia", en los cuales podría corresponder, por ejemplo, mejorar la evaluación de la vía aérea, optimizar precauciones asociadas al uso de fármacos depresores de función ventilatoria, entre otras.

Es nuestro objetivo poder presentar en un futuro próximo el estudio de asociación y/o causalidad entre las variables. A partir del presente registro, descriptivamente destaca que, al alta la menor sobrevida se da en el grupo de paro de causa hemorrágica intraprocedimiento (sólo 13\% de los pacientes de alta, tienen una causa hemorrágica) y la mejor; en el grupo de eventos de causa anestésica, donde $47,4 \%$ de los pacientes de alta, presentan esta causa.

En los casos categorizados como secundarios a la ocurrencia del reflejo de Bezold Jarisch, efectivamente se consideraron otras causas precipitantes de un paro cardiorrespiratorio en presencia de un bloqueo espinal, sin embargo, la clínica de presentación, la respuesta rápida a las maniobras y la total certeza de ausencia de otros factores desencadenantes, hacen de ese mecanismo la opción más probable.

Finalmente, agradecemos la atención con la que el Dr. Ojeda ha leído nuestra comunicación y esperamos poder seguir trabajando de manera de dar respuesta a las interrogantes que quedan sin dilucidar, así como realizar trabajos colaborativos que nos permitan la descripción de escenarios más generales.

Dra. María Mercedes Aguirre C. ${ }^{1}$

${ }^{1}$ Departamento de Anestesiología. Hospital Clínico, Universidad de Chile. Santiago, Chile. 


\section{Referencias}

1. Braz L, Módolo N, Do Nascimento P, Bruschi B, Castiglia Y, Ganem E, et al. Perioperative cardiac arrest: a study of 53 718 anaesthetics over $9 \mathrm{yr}$ from a Brazilian teaching hospital, Br J Anaesth 2006; 96: 596-75.

2. Ellis S, Newland M, Simonson J, Reed Peters K, Romberger D, Mercer D, et al. Anesthesia-related Cardiac Arrest. Anesthesiology 2014; 120: 829-38.

3. Nunnally ME, O'Connor MF, Kordylewski H, Westlake B, Dutton RP. The incidence and risk factors for perioperative cardiac arrest observed in the national anesthesia clinical outcomes registry. Anesth Analg 2015; 120 (2): 364-70.

4. Jacobs I, Nadkarni, Bahr J, Berg RA, Billi JE, Bossaert L, et al. Cardiac arrest and cardiopulmonary resuscitation outcome reports: update and simplification of the Utstein templa- tes for resuscitation registries: a statement for healthcare professionals from a task force of the International Liaison Committee on Resuscitation (American Heart Association, European Resuscitation Council, Australian Resuscitation Council, New Zealand Resuscitation Council, Heart and Stroke Foundation of Canada, InterAmerican Heart Foundation, Resuscitation Councils of Southern Africa). Circulation 2004; 110 (21): 3385-97.

Correspondencia a:

María Mercedes Aguirre C.

Departamento de Anestesiología.

Hospital Clínico Universidad de Chile.

Santos Dumont 999, Independencia, Santiago, Chile.

mmaguirre@uchile.cl 